\title{
Temporal variability predicts the magnitude of between-group attentional blink differences in developmental dyslexia: a meta-analysis
}

Nicholas A Badcock, Joanna C Kidd

Background. Here we report on a meta-analysis of the between-group main effect (Group Difference) noted in the attentional blink $(A B)$ research focussed on specific reading impairment, commonly referred to as developmental dyslexia. The AB effect relates to a limitation in the allocation of attention over time and is examined in a dual-target rapid serial visual presentation (RSVP) paradigm. When the second target appears in close temporal proximity to the first target, the second target is reported less accurately. Method. A Web of Science search with terms "attentional blink" \& dyslexia returned 13 $A B$ experiments (11 papers) conducted with developmental dyslexia. After exclusions, 12 experiments were included in the meta-analysis. The main pattern of performance from those experiments was lower overall accuracy in groups of individuals with dyslexia relative to typically reading peers. That is, a between-group main effect. This metaanalysis examined the size of the Group Difference in relation to temporal and task-set related features, which differed between and within experiments. Results. Random effects modelling indicated a significant Group Difference of $-0.74,[95 \% \mathrm{Cl}:-.96,-.52], \mathrm{p}<$ .001 (excluding one anomalous result): implicating significantly poorer overall dual-target performance in dyslexic readers. Meta-regression analyses indicated two variables related to the Group Difference; pre-RSVP time and temporal variability of the second target relative to the first target within the RSVP. Discussion. It is suggested that the endogenous engagement of the temporal features of task-set is slower or disrupted in developmental dyslexia. 


\section{Title Page}

2 Title: Temporal variability predicts the magnitude of between-group attentional blink differences

3 in developmental dyslexia: a meta-analysis

4 Authors: Nicholas A. Badcock \& Joanna C. Kidd

5 ARC Centre of Excellence in Cognition and its Disorders, Department of Cognitive Science,

6 Macquarie University

7 North Ryde, New South Wales, Australia

8 Corresponding Author: Nicholas A. Badcock

9 Australian Hearing Hub, 16 University Drive

10 North Ryde, New South Wales, Australia, 2109

11 Phone number: +61298504067

12 e-mail: nicholas.badcock@mq.edu.au 


\section{Introduction}

14 Aside from a specific difficulty with the typical acquisition of reading, developmental dyslexia

15 has been associated with a number of cognitive weaknesses. One of these weaknesses is the

16 ability to rapidly deploy attention across time (Tallal, 1976; e.g. Hari \& Renvall, 2001). Here we

17 focus on a single paradigm used to assess the allocation of visual attention across time: a dual-

18 target Rapid Serial Visual Presentation (RSVP) paradigm. Performance in this paradigm has

19 been described as an 'Attentional Blink' (AB). The $\mathrm{AB}$ is an attentional phenomenon whereby the

20 processing of the first target (T1) is considered to disrupt the processing of a second target (T2)

21 when the two targets appear in close temporal proximity (i.e. within $500 \mathrm{~ms}$; Broadbent \&

22 Broadbent, 1987; Raymond, Shapiro \& Arnell, 1992). The standard AB pattern is illustrated by

23 the solid line in Fig 1. The main point to note is that, at short inter-target intervals (e.g. 200 to 300

$24 \mathrm{~ms}$ ), T2 accuracy is lower than at long (e.g. 500 to $700 \mathrm{~ms}$ ) intervals.

Insert Fig 1 about here

26 In a review of the $\mathrm{AB}$ literature on developmental dyslexia, McLean, Castles, Coltheart, and

27 Stuart (2010) demonstrated that the most common difference between dyslexic and typically

28 reading groups was a main effect; that is, overall, the performance of the dyslexic readers was

29 lower than for typical readers. Therefore, rather than a difficulty in rapidly deploying attention

30 across time (or "sluggish attentional shifting", see Hari \& Renvall, 2001), dyslexic readers had a

31 general difficulty with the $\mathrm{AB}$ paradigm. To illustrate this point, four different patterns of $\mathrm{AB}$

32 performance are presented in Fig 1. Specific difficulties with the AB may relate to deeper, longer,

33 or deeper and longer effects (illustrated in Fig 1, panels A to C). However, what is noted in the

34 dyslexia literature is the fourth option, lower overall accuracy (see Fig 1, panel D), reflecting a

35 general difficulty with the dual-target paradigm (note: unless otherwise specified, references to 
36 'single-target' and 'dual-target' refer to RSVP procedures). The current paper reports on a meta-

37 analysis of the $\mathrm{AB}$ and dyslexia literature to explore this general difficulty.

38 The $\mathrm{AB}$ is considered to reflect a temporal limitation of attention. Most generally it is thought

39 that a finite set of resources is required to consolidate a short-term representation of a target item

40 for conscious report. While these resources are consolidating of T1, the representation of T2 may

41 decay and will not be available for conscious report (for reviews see Dux \& Marois, 2009;

42 Martens \& Wyble, 2010). If this consolidation lasts longer, short-term representations waiting to

43 be processed may decay; in order to update these representations for consolidation, regressive

44 eye-movements made during reading may increase: although regressions may have other

45 explanations as well, e.g. checking for comprehension. This pattern of eye-movements has been

46 noted in developmental dyslexia (Schneps et al., 2013). However, of the thirteen experiments

47 published in this area, only three identify $\mathrm{AB}$ performance consistent with longer consolidation

48 (or more rapid memory decay). These three experiments (Hari, Valta \& Uutela, 1999; Lallier et

49 al., 2010; Laasonen et al., 2012) indicate that the accuracy of T2 report in dyslexia reaches the

50 same level of accuracy as that of typical readers at long inter-target intervals; however, the time

51 required to reach this accuracy is longer for dyslexic than typical readers. The majority of the

52 experiments do not demonstrate this interaction, with accuracy remaining lower in dyslexic

53 readers across all inter-target intervals (Visser, Boden \& Giaschi, 2004; Buchholz \& Aimola

54 Davies, 2007; Badcock, Hogben \& Fletcher, 2008; Facoetti et al., 2008; Lallier, Donnadieu \&

55 Valdois, 2010; McLean et al., 2010). One anomalous experiment reports higher accuracy across

56 all inter-target intervals in a group of dyslexic readers (Lacroix et al., 2005): this will be

57 considered in the discussion. If the majority of the evidence points to a general deficit in the $\mathrm{AB}$

58 paradigm for dyslexic readers, what underpins the general deficit? 
59 When McLean et al. (2010) added single-target accuracy as a covariate in their analysis, twenty

60 per cent of the dual-target performance difference between dyslexic and typical readers was

61 accounted for. When controlling for single-target accuracy, in combination with a continuous-

62 performance measure (accounting for nine per cent of the between-group variation), the between-

63 group effect was no longer significant. Simulating this sort of inattention factor produces patterns

64 of data that mimic dyslexic group performance (Stuart, McAnally \& Castles, 2001; Roach,

65 Edwards \& Hogben, 2004). A general factor common to single- and dual-target paradigms may

66 account for the between group differences noted in the $\mathrm{AB}$ and also have much broader

67 implications beyond RSVP paradigms.

68 In RSVP paradigms, the target serial position varies in time relative to the onset of the RSVP.

69 Single-target performance has been shown to be sensitive to this temporal variation. In a series of 70 experiments, Ariga and Yokosawa (2008) demonstrated that single-target accuracy increased as a

71 function of foreperiod: that is, at longer intervals from the onset of the RSVP, accuracy was

72 higher. There is a body of literature on the effects of temporal orienting, particularly with respect

73 to reaction time (Niemi \& Näätänen, 1981; Nobre, Correa \& Coull, 2007), but temporal

74 predictability and cueing have also been demonstrated to increase accuracy in the $\mathrm{AB}$ (cueing

75 Martens \& Johnson, 2005; predictability Badcock et al., 2013). Recently, Tang, Badcock, and

76 Visser (2013) demonstrated that dual-target practice improvements are attributable to learning the

77 target temporal locations. Given that temporal orienting has a role in both single- and dual-

78 targets, it presents as a candidate explanation for differences between dyslexic and typical readers

79 in RSVP performance.

80 Another element common to single- and dual-target paradigms is the engagement of 'task-set'.

81 Monsell proposed the concept of task-set with respect to task-switching paradigms (Rogers \&

82 Monsell, 1995; Monsell, 1996) whereby, even during a simple paradigm when the task was well 
83 known to the participants, a cognitive model of the task-requirements must be engaged to

84 complete the task. In an RSVP paradigm, the cognitive model includes searching for letters while

85 ignoring numbers and reporting the identity of letters. Therefore, this concept is similar to the

86 proposal that endogenous control of a 'visual-filter' is set up at the outset of the RSVP task (Di

87 Lollo et al., 2005). Di Lollo et al. suggest that the AB is caused by a 'temporal loss of control' of

88 the visual filter (but see Dell'Acqua et al., 2009; and also Olivers et al., 2011). The engagement

89 of task-set may account for time-related increases in single-target accuracy (i.e. Ariga \&

90 Yokosawa, 2008). Nevertheless, it is an additional candidate for the differences between dyslexic

91 and typical readers noted in RSVP performance.

92 The current investigation aimed to explore the basis of the significantly lower dual-target

93 accuracy that has been reported in dyslexic versus typical reading groups. Based on evidence that

94 single-target performance can account for the dual-target between-group difference, we examined

95 variability in temporal and task-set related features via a meta-analysis of the $\mathrm{AB}$ literature on

96 developmental dyslexia. Temporal variations and task-set requirements were selected as common

97 to both single- and dual-target tasks.

\section{Method}

99 Experiment Selection

100 Searching the Web of Science for "attentional blink" \& dyslexia returned 26 entries (23rd of

101 September 2014). Papers were required to include a comparison of dyslexic readers with respect

102 to age-matched typical readers on a dual-target task requiring the identification and/or detection

103 of two targets: 12 papers were relevant, two of which included two experiments (Visser, Boden \&

104 Giaschi, 2004; Buchholz \& Aimola Davies, 2007). Badcock et al. (2011) report a reanalysis of

105 their 2008 data. This was not included in the present meta-analysis because it is not an 
106 independent experiment. McLean et al. (2011) report an extension of their (2010) research,

107 doubling the sample size. The 2010 work was therefore excluded from the analyses. The

108 comprehensiveness of this set of experiments was confirmed using PubMed and PsycInfo using

109 the same search terms, which returned the same set of experiments.

110 The final number of experiments included was 12 (Hari, Valta \& Uutela, 1999; Experiments 1

111 and 2, Visser, Boden \& Giaschi, 2004; Lacroix et al., 2005; Experiment 1 and 2, Buchholz \&

112 Aimola Davies, 2007; Badcock, Hogben \& Fletcher, 2008; Facoetti et al., 2008; Lallier et al.,

113 2010; Lallier, Donnadieu \& Valdois, 2010; McLean et al., 2011; Laasonen et al., 2012).

114 As in any field, a bias for the publication of significant results may mean that this meta-analysis

115 overestimates the true between-group difference (i.e. "the file drawer problem" Rosenthal, 1979).

116 However, as the $\mathrm{AB}$ and dyslexia research has focussed on a between-group interaction, bias in

117 not reporting the between-group main effect should be reduced. The main objective of this

118 analysis is to examine variables related to this between-group difference. Noteworthy

119 relationships will need to be directly manipulated in dyslexia investigations prior to theoretical

120 incorporation. This meta-analysis takes the additional step of exploring the meta-analytic variable

121 with respect to task-parameters. The PRISMA checklist (Moher et al., 2009) is included in the

122 Supplementary Materials.

123 Variable selection and calculation

124 In addition to the Group difference, seven variables were included as possible moderators: pre-

125 RSVP onset time; stimulus onset asynchrony; T1, T2, and RSVP temporal variation, total target

126 and distractor identities, and task complexity.. All data points for each study, including McLean et

127 al. (2010), are included in Table 1. 


\section{Group difference}

129 The Group Difference was estimated as the effect size of between-group difference (dyslexic

130 minus typical) for overall mean $\mathrm{T} 2$ accuracy, given that $\mathrm{T} 1$ was correctly reported (T2|T1). This

131 is the average accuracy at each of the inter-target intervals. The associated variance, required for

132 the meta-analysis, was the standard deviation of this accuracy at each inter-target interval. The

133 Group Difference was calculated in standard deviation units, Cohen's d, using an unbiased

134 estimation (Cumming, 2012, 2013) with the 'escalc' function in 'metafor' (Viechtbauer, 2010). In

135 all but two cases, the mean and standard deviation for the dyslexic and control reading groups

136 were supplied by authors, if it was not available in the published manuscripts (available in Lallier

137 et al., 2010). Where the required data were not reported or could not be obtained (Hari, Valta \&

138 Uutela, 1999; Lacroix et al., 2005), the data were estimated from the published figures using

139 WebPlotDigitizer (Rohatgi, 2014).

140 Pre-RSVP onset time

141 Pre-RSVP onset time is defined as the time period (in ms) before the presentation of the first

142 RSVP item, typically constituting the inter-trial interval. This includes the duration of a fixation

143 symbol and any fixation to RSVP onset time where no stimulus was presented. In three

144 experiments (Visser, Boden \& Giaschi, 2004; Laasonen et al., 2012), the fixation symbol

145 remained on screen until a key-press. These experiments were excluded on the basis that the

146 presentation duration of the fixation could not be determined. Hari et al.'s (1999) experiment

147 presented no fixation symbol, therefore, forming a baseline condition where the first element of

148 the presentation is a distracter item. The final number of experiments included for this variable 
149 was eight, after the Lacroix et al. (2005) experiment was excluded based on the test of

150 homogeneity (see results).

\section{Stimulus onset asynchrony (SOA)}

152 SOA represents the time period between the onset of one stimulus and the next. This was

153 determined from the method sections of the respective papers.

\section{$154 T 1, T 2$, and RSVP temporal variation}

155 Temporal variation was calculated as the difference between the minimum and maximum

156 temporal presentation values of T1 within the RSVP and T2 relative to T1, and the longest minus

157 the shortest RSVP sequences.

158 For example, if $\mathrm{T} 1$ is presented at RSVP positions 6,7 , and 8 , and the stimulus onset asynchrony

159 is $100 \mathrm{~ms}$, this would correspond to 600 (minimum), 700, and 800 (maximum) ms, with a

160 difference of $200 \mathrm{~ms}$. If T2 is presented at positions 1 to 12 following T1, this would correspond

161 to 100 through to 1200 , with a difference of $1100 \mathrm{~ms}$. If two distractor items always follow T2 in

162 the RSVP, then the minimum RSVP would be $900(\min \mathrm{T} 1+\min \mathrm{T} 2+2=600+100+200)$, the

163 maximum $2200(\max T 1+\max T 2+200=800+1200+200)$, with a difference of $1300 \mathrm{~ms}$.

164 In the case of Facoetti et al. (2008) where only two targets and accompanying masks were

165 presented, target and RSVP timings corresponds to the number of temporal positions starting with

166 the earliest time of $\mathrm{T} 1$ presentation following fixation $(125 \mathrm{~ms})$. It is worth noting that this

167 'skeletal' RSVP paradigm may produce more variable results at the electrophysiological level

168 (see Craston, Wyble \& Bowman, 2006). 
170 By 'identity' we refer to the number of standard verbal labels of T1, T2, and distractors. For

171 example, if T2 is a fixed letter of the alphabet (e.g. letter X), and T1 as well as the distractors are

172 any letter other than that used for T2, there is 1 identity for T2, 25 possible identities for $\mathrm{T} 1$, and

17325 for the distractors: with the total number of identities being 26 . Note: T1 and the distractor

174 identities both have 25 possibilities because they are randomly selected on each trial and the T1

175 identity for one trial will be the distractor identity for another trial. This example is for illustrative

176 purposes, many studies omit 'I', 'O', and 'Q' due to the limited masking properties.

177 The Visser et al. (2004) and McLean et al. (2011) experiments included random-dot distractors

178 with different 'identities' for each presentation. Although the precise number of identities is

179 difficult to determine, this was classified as a single identity given that independent semantic

180 labels could not be applied to these variations.

\section{Task complexity}

182 Task complexity was calculated as the sum of following criteria. 1) A switch between T1 and T2.

183 This included a change in decision type (identification to detection), a change in stimulus search

184 (e.g., any white letter to a black letter X), a change in stimulus category (e.g. arrows to shapes),

185 or a change in spatial location. 2) Whether the target and distractor items were from the same

186 stimulus category. One point was assigned for each of the five criteria that were met. Analysis

187 The between-group effect-size (i.e. Group Difference) across studies was estimated using a

188 random effect model implemented in R using the 'metafor' package (Viechtbauer, 2010). The

189 relationships between the dependent measures were tested using point estimate and rank

190 correlations (Pearson's product-moment and Spearman's rho) and moderation was tested using 
191 random effects modelling. Both parametric and non-parametric correlations are reported to be

192 conservative with respect to the small number of studies.

\section{Not correcting for multiple comparisons}

194 Correlations were calculated to examine the relationships between the dependent measures in this

195 paper. We did not correct for multiple comparisons in this instance. This decision was made to

196 reduce the risk of Type 2 errors and on the basis that relationships of note in the meta-analysis

197 will need to be demonstrated empirically before they are applied theoretically (see Cabin \&

198 Mitchell, 2000, for a discussion on considering correcting for multiple comparisons).

\section{Results}

200 The Group Difference for each study is presented in a forest plot in Fig 2. The overall difference

201 was $-0.70, \mathrm{p}=0.001$, indicating that across the 12 experiments, overall $\mathrm{T} 2 \mid \mathrm{T} 1$ accuracy was

202 poorer in dyslexic readers. A test of heterogeneity indicated that $57 \%$ of the variation was due to

203 between study differences, $Q_{(11)}=23.6, p=.015$. This was entirely attributable to the Lacroix et

204 al. (2005) experiment, its exclusion leading to a non-significant test for heterogeneity: $0 \%, Q_{(10)}=$

$2059.8, \mathrm{p}=.46$. Thus the Lacroix et al. result is sufficiently different that it may be considered

206 sampled from a separate population. For this reason, it was excluded from subsequent analyses.

207 The overall estimate of effect size excluding this experiment was -0.74 , [95\% CI: $-.96,-.52], \mathrm{p}<$.

208001.

209

Insert Fig $2 \&$ Table 1 about here

210 Descriptive statistics for the 8 variables are presented in Table 1 (data points for all experiments

211 are also presented). The SOA, T1 and T2 time difference, and total identities variables were not

212 normally distributed. Therefore, Spearman non-parametric correlations are more appropriate 
213 indices of the relationships for these variables, and have been reported along side Pearson

214 product-moment values in Table 2. Most critical is the bottom row in which correlations for the

215 relationship of Group Difference to all other variables are reported. There are three relationships

216 worth drawing attention to. The first is a strong negative relationship between Pre-RSVP time and

217 the Group Difference (Pearson $=-.66$, Spearman $=-.86$ ), indicating that the greater the time

218 before the onset of the RSVP, the greater the between-group accuracy discrepancy. The second

219 two are the strong positive correlations between T2 time difference relative to T1 (Pearson $=.62$,

220 Spearman $=.68)$ and the RSVP time difference $($ Pearson $=.58$, Spearman $=.62)$ and the Group

221 Difference. These relationships indicate that the greater the temporal variability of $\mathrm{T} 2$ relative to

$222 \mathrm{~T} 1$ and within the overall RSVP, the smaller the between-group difference. It is important to note

223 that all three of these variables are highly correlated with absolute magnitudes between .68 and .

224 88. The relationships between the Group Difference and Pre-RSVP time and T2 time difference

225 relative to $\mathrm{T} 1$ are presented in scatter plots with linear fits in Fig 3. The relationship between the

226 Group Difference and Pre-RSVP time was the only significant moderating relationship: $\beta=$

$227-0.001[-0.002,0.000], \mathrm{Q}_{(1)}=3.87, \mathrm{p}=0.049$. This indicates that a $1 \mathrm{msec}$ increase in pre-RSVP

228 time increases the group difference by 0.001 standard deviations units; an additional $500 \mathrm{msec}$

229 before RSVP onset increases the difference by 0.5 standard deviation units. However, for the

230 relationship between the Group Difference and T2 time difference relative to T1, whilst not

231 statically significant, the $80 \%$ confidence intervals did not overlap with zero, $\beta=0.0005[80 \%$

$232 \mathrm{CI} ; 0.000,0.001], \mathrm{Q}_{(1)}=1.65, \mathrm{p}=0.20$. This indicates that a $1 \mathrm{msec}$ increase in $\mathrm{T} 2$ time difference

233 decreases the group difference by 0.0005 standard deviations units; the addition of $500 \mathrm{msec}$ of

234 variability decreases the difference by 0.25 standard deviation units. Considering the exploratory

235 nature of moderator variables in a meta-analysis, we consider this to be suitable for further

236 consideration (Cumming, 2012). 


\section{Discussion}

239 In a meta-analysis of attentional blink (AB) experiments focussed on developmental dyslexia, we

240 examined whether the overall between-group difference in T2 accuracy was related to variability

241 in temporal and task-set related features. As noted by McLean et al. (2010), the clearest pattern in

242 this literature is that performance of groups of individuals with dyslexia is poorer overall (see Fig

2431 panel D); that is, statistically, there is a main effect, indicative of a general difficulty with the

244 dual-target rapid serial visual presentation (RSVP) paradigm in dyslexia, rather than a specific

$245 \mathrm{AB}$ effect. The results of the meta-analysis support this suggestion, with a group difference

246 between -0.70 and -0.74 standard deviation units (see Fig 2 for a forest plot). Using correlation

247 and moderator linear modelling, pre-RSVP time and T2 temporal variability were identified as

248 explanatory, presentation-related factors for the group difference.

249 Pre-RSVP time and endogenous control

250 The longer the time period before the RSVP onset, the greater the difference between-groups. To

251 be clear, three of the $\mathrm{AB}$ and dyslexia experiments included in the meta-analysis were excluded

252 from the analysis of pre-RSVP time because the fixation symbol remained onscreen until a key-

253 press. Therefore, the finding is limited as it is based on eight experiments, with pre-RSVP times

254 of $0,500,600$, and $1000 \mathrm{~ms}$. However, in support of the pattern, we have conducted two pilot

255 studies with adults, unselected for reading ability, in which we manipulated the duration of the

256 pre-RSVP time and found that this influences overall performance: shorter intervals

257 corresponding with lower accuracy. Given the strength of the relationship and the results of the

258 pilot studies, pre-RSVP time is worth further consideration. The pre-RSVP time may have a

259 preparatory function for the engagement of task-set. Task-set is a cognitive model of the task 
260 requirements (Rogers \& Monsell, 1995; Monsell, 1996). This is a similar concept to the visual

261 filter which is implicated in selection theories of the AB (e.g. Di Lollo et al., 2005). For a task

262 including two number targets in a series of black letter distractors, the task-set would involve

263 ignoring letters and attending to numbers.

264 The task-set may also incorporate goals. Ferlazzo et al. demonstrated that the AB could be

265 mediated by varying the instructions to participants (Ferlazzo et al., 2007). With standard

266 instructions, e.g. report the identity of two numbers, an $\mathrm{AB}$ effect was observed. However, with

267 modified instructions consisting of a single goal, e.g. report the sum of the numbers, the $\mathrm{AB}$

268 effect was not observed. In relation to temporal effects, the engagement time of task-set may be

269 shorter for a single goal, resulting in more accurate performance.

270 Temporal expectations of target presentation may be part of task-set with respect to perceptual

271 expectations. When attention can be directed to a particular point in time, decisions regarding a

272 target are more accurate (again see Nobre, Correa \& Coull, 2007, for a revew). This has also been

273 demonstrated in the AB. When Martens and Johnson (2005) cued the temporal location of targets

274 within an RSVP sequence, the $\mathrm{AB}$ was reduced. Similar reductions were found when Badcock et

275 al. (2013) individually tailored the time before T1 and made it predictable between trials; relative

276 to when the temporal location of T1 was randomly varied between 250 and $750 \mathrm{~ms}$, the $\mathrm{AB}$ was

277 reduced. Further to this, Tang, Badcock, and Visser (2013) demonstrated that practice with the

$278 \mathrm{AB}$ task increased expectations about the temporal locations of the targets. This suggests that,

279 with exposure to RSVP tasks, observers are implicitly generating expectations about the timing of

280 the presentation. This may be a longer learning process in dyslexia (Badcock, Hogben \& Fletcher,

281 2011), contributing to lower overall accuracy in the AB. 
282 With respect to the engagement of task-set in dyslexia, the pattern of overall group differences in

283 the $\mathrm{AB}$ can be accounted for by a specific difficulty with the endogenous engagement of task-set.

284 Endogenous engagement relates to internally- or observer-driven behaviour, as opposed to

285 exogenous engagement, which relates to stimulus-driven behaviour. The engagement of task-set

286 prior to the RSVP onset requires endogenous control. With limited or no pre-RSVP time, neither

287 group can prepare and the typical readers do not gain the advantage that they do at longer pre-

288 RSVP times.

289 Through the endogenous engagement of task-set, increasing the pre-RSVP time should enhance

290 preparation for the task and result in better performance (as evidenced by Badcock et al., 2013).

291 This was observed in the current data, examining the correlation between overall T2 accuracy and

292 pre-RSVP time (Spearman, single-tailed), but was only reliable in the typical readers; rho $=0.71$,

$293 \mathrm{p}=0.02$; dyslexic readers; rho $=0.22, \mathrm{p}=0.30$; consistent with the proposed difficulty with the

294 endogenous engagement of task-set in dyslexic readers. It is important to note that this

295 relationship relates to temporal features of task-set and not to target and distracter categories

296 (Maki \& Padmanabhan, 1994; Maki et al., 1997) or task complexities such as task-switches (see

297 Potter et al., 1998), which were not related the group difference for the current data. Experimental

298 manipulation of these factors should be conducted before it can be confidently concluded that

299 categories and complexities have no effect.

300 T2 timing and endogenous or exogenous control

301 Greater temporal variability of $\mathrm{T} 2$ relative to $\mathrm{T} 1$ was associated with smaller group differences in

302 overall T2 accuracy. This variable was also correlated with the variability of the RSVP duration

303 (.73 to .88$)$ as well as the pre-RSVP time (-.77 to -.87$)$. It was calculated as the difference

304 between the maximum and minimum temporal positions of T2 relative to T1. As evident in the 
305 preprint of this article (Badcock \& Kidd, 2014), this variable is highly correlated with the

306 minimum (0.73) and maximum (0.99) T2 temporal positions, so the greater variability is not

307 independent of longer time periods before the presentation of $\mathrm{T} 2$. Therefore, this can be

308 considered as T2 timing.

309 With respect to T2 timing, the engagement of task-set following the RSVP onset can be driven

310 endogenously but will be exogenously influenced by the stimulus, essentially reminding the

311 observer of task requirements. Through the exogenous engagement of task-set, increasing the

312 time before the presentation of a target should enhance preparation for the task and result in

313 better performance (e.g., Ariga \& Yokosawa, 2008). However, the opposite pattern was observed

314 in the current data: the correlation between overall $\mathrm{T} 2$ accuracy and maximum $\mathrm{T} 2$ temporal

315 position within the RSVP was negative, Spearman's rho $=-0.56, p=0.07$. This does not converge

316 with the results for the group difference, suggesting that temporal variability may underpin the

317 relationship between $\mathrm{T} 2$ timing and the group difference.

318 As mentioned, temporal expectations are part of task-set and anticipating when a stimulus will

319 occur improves accuracy (for a review see Nobre, Correa \& Coull, 2007). With respect to

320 temporal variability, as variability increases, temporal orienting would be poorer, resulting in

321 lower accuracy. This was observed in the current data for both groups, the correlation between

322 overall T2 accuracy and the maximum minus minimum T2 temporal position within the RSVP,

323 (Spearman, single-tailed), but was only reliable in the typical readers; rho $=-0.69, \mathrm{p}=0.01$;

324 dyslexic readers; rho $=-0.40, p=0.10$. These results are consistent with a performance advantage

325 for typical readers for presentations with small temporal variability, whereas this does not seem to

326 be the case for dyslexic readers. This advantage may be underpinned or further exaggerated by

327 preparation gained during the pre-RSVP interval. 
328

Perceptual learning

329 This interpretation of the findings implicates endogenous control difficulties with dyslexia. To

330 reiterate, this includes poor or slow task preparation and poor or slow tuning of temporal

331 attention to target timings. Perceptual learning, specifically with respect to the Perceptual

332 Anchoring Theory of dyslexia (Ahissar, 2007), also offers an account for some aspects of the

333 current findings. A sound perceptual learning system allows observers to take advantage of

334 regularities in psychophysical presentations. By forming 'perceptual anchors' to these

335 regularities, performance improves as the task processing switches from being effortful to

336 automatic. For the current results, repetition of the pre-RSVP time or small temporal variability in

337 T2 position may have been utilised by typical observers to improve accuracy, whereas this is not

338 the case in dyslexia (but see Ziegler, 2008). An anchoring deficit in dyslexia would account for

339 limited performance improvement with increasing pre-RSVP times, as dyslexic readers would not

340 form an anchor to this regularity. Similarly, dyslexic readers may not take advantage of small

341 temporal variability in T2 position, whereas it may be difficult for any observer, sound anchoring

342 or otherwise, to anchor to larger temporal variability, as was observed in the typical readers. We

343 do note that perceptual anchoring does not account for the increasing group difference with

344 increasing pre-RSVP time (e.g., a preparatory component); however, the methods and theories

345 relevant to perceptual anchoring may prove useful in investigating the current suggestions.

346 A major caveat to the above suggestions is that meta-analytic techniques stepping beyond tests of

347 homogeneity are exploratory in nature. The variables have been highlighted within inherently

348 complex paradigms conducted in multiple laboratories each with subtly different goals in mind.

349 Therefore we raise these variables, and subsequent discussion, as speculation that requires

350 empirical interrogation. 


\section{Further considerations}

352 The Lacroix et al. anomalous result

353 There is one anomalous result in the $\mathrm{AB}$ and dyslexia literature. Lacroix et al. (2005) reported

354 better overall performance in a group of children (15-years of age) with developmental dyslexia,

355 relatively to typically reading peers. The task involved the identification of red number targets in

356 black number distracters. Buchholz and Aimola Davies (2007) conducted the same design in

357 adults and found the typical pattern of results. Lallier et al. (2010) conducted a T1 identification-

358 T2 detection variation in younger children (approximately 10-years of age) and also found the

359 typical pattern of results. One feature of the Lacroix et al. results that stands out is that the overall

360 T2 accuracy (i.e. the mean of the two groups) was low (49\% with next lowest, $73 \%$ ). When

361 considered as a predictor of the Group Difference including the Lacroix et al. result, overall T2

362 accuracy was a significant moderator; $\beta=-4.41,[-7.01,-1.80], \mathrm{Q}_{(1)}=10.99, \mathrm{p}<0.001$ (see Fig $3634)$.

365 It is possible that at this level of difficulty, observers may adopt a different strategy, which may

366 not necessarily be conscious. Lacroix et al. (2005) suggest that superior dual-target accuracy in

367 the dyslexic group may be explained by development differences in the depth of target

368 processing. Suppose the group with dyslexia have less detailed representations for numbers and

369 the group with typical reading have more detailed representations for numbers. If greater

370 resources were required to access the more detailed representations, the typical readers would

371 exhibit poorer performance. In order to best integrate this result with the other literature,

372 replication reducing overall accuracy may be an important step. 
373 Confounded variables

374 There were significant inter-correlations between the variables, which are due to the

375 commonalities and differences between experiments. For example, pre-RSVP time and total

376 identities were correlated with T2 temporal variability. These are impossible to disentangle in the

377 current dataset. Without direct experimental manipulation of these variables, it cannot be

378 determined whether the highlighted variables are critical factors. Therefore we have purposefully

379 restricted the considerations of the relationship between the highlighted variables and reading

380 mechanisms until such a relationship is demonstrated.

381 One suggestion arising from these confounds is that a common paradigm be adopted for $\mathrm{AB}$ and

382 dyslexia research. Considering the limited reliance on literacy experience for shape-targets and

383 random-dot distracters used by McLean et al. (2010) and Visser et al. (2004), this seems like a

384 good starting place. Furthermore, this paradigm has also been used to evaluate the relationship

385 between AB performance and typical reading (McLean et al., 2009; La Rocque \& Visser, 2009),

386 so there is a growing body of evidence common to this target and distracter set related to reading.

387 Subtypes of dyslexia

388 We have not been able to address subtypes of dyslexia (Jones, Castles \& Kohnen, 2011; Coltheart

$389 \&$ Kohnen, 2012) in this meta-analysis and we want to flag this as an important consideration for

390 future research. McLean et al. (2010) examined their results with respect to non-word and

391 irregular word reading independently but did not find a difference with respect to the between

392 group relationship. La Rocque and Visser (2009) found a relationship between non-wording

393 reading and $\mathrm{AB}$ magnitude in typically reading adults, suggesting some role of phonological

394 processing. However, this specifically related to a between group interaction rather than an

395 overall main effect, therefore it is not clear what role phonological processing would have for the 
396 current set of variables. The main effect is also evident in shallow orthographies (i.e. Italian,

397 Facoetti et al., 2008) in which the letter-sound correspondences tend not to underpin reading

398 difficulties; therefore, exploration of this relationship should not be limited to phonological

399 deficits, and attentional subtypes (Friedmann, Kerbel \& Shvimer, 2010; Kohnen et al., 2012) and

400 capacities (Valdois et al., 2003; Bosse, Tainturier \& Valdois, 2007) should also be examined. A

401 critical step in pinning down the relevance of any variable in relationship to dyslexia, is pinning

402 down which particular component of the reading process, and in turn which subtype, the variable

403 is associated with.

404 The available evidence

405 This research is limited by the available evidence. The number of experiments published in the

406 literature is small, and exclusory criteria reduced this to 11 ( 8 for one variable) in the current

407 meta-analysis. The dataset is limited to published work which may be associated with biases with

408 respect to the publication of significant effects. The results should be interpreted with these

409 limitations in mind.

\section{Summary and Conclusion}

411 In this paper we report on a meta-analysis of published $\mathrm{AB}$ experiments conducted in

412 developmental dyslexia. We examined the common occurrence of an overall between-group

413 difference where dyslexic readers exhibit lower target reporting accuracy in relation to task

414 parameters which varied between and within experiments. This between-group difference was

415 related to pre-RSVP time and second target temporal variablity. Future investigations should

416 consider the endogenous engagement of task-set and temporal learning in the $\mathrm{AB}$ and dyslexia in

417 order to best determine the relationship between reading and the $A B$, and whether it may be a

418 potential tool for intervention. 


\section{Acknowledgements}

420 The authors would like to thank Veronika Coltheart for her comments on an earlier version of the

421 manuscript, Gen McArthur for her valuable input and support, as well as Sachiko Kinoshita and

422 Maree Tyson-Parry for their ears and thought provoking discussions. We would also like to thank

423 all the authors of the $\mathrm{AB}$ and dyslexia experiment for sharing their data and details of their

424 research procedures. 
426 Ahissar M. 2007. Dyslexia and the anchoring-deficit hypothesis. Trends in Cognitive Sciences $427 \quad 11: 458-465$.

428 Ariga A, Yokosawa K. 2008. Attentional awakening: gradual modulation of temporal attention in 429 rapid serial visual presentation. Psychological Research 72:192-202.

430 Badcock NA, Badcock DR, Fletcher J, Hogben J. 2013. The role of preparation time in the 431 attentional blink. Vision Research 76:68-76.

432 Badcock NA, Hogben JH, Fletcher JF. 2008. No differential attentional blink in dyslexia after 433 controlling for baseline sensitivity. Vision Research 48:1497-1502.

434 Badcock NA, Hogben JH, Fletcher JF. 2011. Dyslexia and practice in the attentional blink:

435 Evidence of slower task learning in dyslexia. Cortex 47:494-500.

436 Badcock NA, Kidd JC. 2014. Temporal variability predicts the magnitude of between-group attentional blink differences in developmental dyslexia: a meta-analysis. PeerJ PrePrints

Bosse M-L, Tainturier MJ, Valdois S. 2007. Developmental dyslexia: The visual attention span 2:e310v2.

441 Broadbent DE, Broadbent MH. 1987. From detection to identification: response to multiple deficit hypothesis. Cognition 104:198-230.

443 Buchholz J, Aimola Davies A. 2007. Attentional blink deficits observed in dyslexia depend on $444 \quad$ task demands. Vision Research 47:1292-1302.

445 Cabin RJ, Mitchell RJ. 2000. To Bonferroni or Not to Bonferroni: When and How Are the 446 Questions. Bulletin of the Ecological Society of America 81:246-248. 
447 Coltheart M, Kohnen S. 2012. Acquired and developmental disorders of reading and spelling. In:

$448 \quad$ Faust M ed. The Handbook of the Neuropsychology of Language. Chicester, UK: Wiley-

449 Blackwell Publishers, 892-920).

450 Craston P, Wyble B, Bowman H. 2006. An EEG study of masking effects in RSVP. Journal of $451 \quad$ Vision 6:1016.

452 Cumming G. 2012. Understanding The New Statistics: Effect Sizes, Confidence Intervals, and $453 \quad$ Meta-Analysis. New York: Routledge.

454 Cumming G. 2013. The New Statistics Why and How. Psychological $455 \quad$ Science:0956797613504966.

456 Dell'Acqua R, Jolicoeur P, Luria R, Pluchino P. 2009. Reevaluating encoding-capacity limitations 457 as a cause of the attentional blink. Journal of Experimental Psychology. Human

458 Perception and Performance 35:338-351.

459

460

Dux PE, Marois R. 2009. The attentional blink: a review of data and theory. Attention, Perception

461 Facoetti A, Ruffino M, Peru A, Paganoni P, Chelazzi L. 2008. Sluggish engagement and

462 disengagement of non-spatial attention in dyslexic children. Cortex 44:1221-1233.

463 Ferlazzo F, Lucido S, Di Nocera F, Fagioli S, Sdoia S. 2007. Switching Between Goals Mediates 464 the Attentional Blink Effect. Experimental Psychology 54:89-98.

465 Friedmann N, Kerbel N, Shvimer L. 2010. Developmental attentional dyslexia. Cortex 46:1216466 1237.

467 Hari R, Renvall H. 2001. Impaired processing of rapid stimulus sequences in dyslexia. Trends in $468 \quad$ Cognitive Sciences 5:525-532. 
469 Hari R, Valta M, Uutela K. 1999. Prolonged attentional dwell time in dyslexic adults.

$470 \quad$ Neuroscience Letters 271:202-204.

471 Jones K, Castles A, Kohnen S. 2011. Subtypes of developmental reading disorders: Recent

472 developments and directions for treatment. ACQuiring Knowledge in Speech, Language

$473 \quad$ and Hearing 13:79-83.

474 Kohnen S, Nickels L, Castles A, Friedmann N, McArthur G. 2012. When “slime” becomes

475 "smile": Developmental letter position dyslexia in English. Neuropsychologia 50:3681-

$476 \quad 6692$.

477 Laasonen M, Salomaa J, Cousineau D, Leppämäki S, Tani P, Hokkanen L, Dye M. 2012. Project

478 DyAdd: Visual attention in adult dyslexia and ADHD. Brain and Cognition 80:311-327.

479 Lacroix GL, Constantinescu I, Cousineau D, de Almeida RG, Segalowitz N, Grünau M von.

480 2005. Attentional blink differences between adolescent dyslexic and normal readers.

$481 \quad$ Brain and Cognition 57:115-119.

482 Lallier M, Donnadieu S, Berger C, Valdois S. 2010. A case study of developmental phonological

483 dyslexia: Is the attentional deficit in the perception of rapid stimuli sequences amodal?

$484 \quad$ Cortex 46:231-241.

485 Lallier M, Donnadieu S, Valdois S. 2010. Visual attentional blink in dyslexic children:

486 Parameterizing the deficit. Vision Research 50:1855-1861.

487 Di Lollo V, Kawahara J, Shahab Ghorashi SM, Enns JT. 2005. The attentional blink: Resource 488 depletion or temporary loss of control? Psychological Research 69:191-200.

489 Maki WS, Couture T, Frigen K, Lien D. 1997. Sources of the attentional blink during rapid serial 490 visual presentation: Perceptual interference and retrieval competition. Journal of

491 Experimental Psychology: Human Perception and Performance 23:1393-1411. 
492 Maki WS, Padmanabhan G. 1994. Transient suppression of processing during rapid serial visual 493 presentation: Acquired distinctiveness of probes modulates the attentional blink. $494 \quad$ Psychonomic Bulletin \& Review 1:499-504.

495 Martens S, Johnson A. 2005. Timing attention: cuing target onset interval attenuates the 496 attentional blink. Memory \& Cognition 33:234-240.

497 Martens S, Wyble B. 2010. The attentional blink: Past, present, and future of a blind spot in 498 perceptual awareness. Neuroscience \& Biobehavioral Reviews 34:947-957.

499 McLean GMT, Castles A, Coltheart V, Stuart GW. 2010. No evidence for a prolonged attentional $500 \quad$ blink in developmental dyslexia. Cortex 46:1317-1329.

501 McLean GMT, Stuart GW, Coltheart V, Castles A. 2011. Visual temporal processing in dyslexia 502 and the magnocellular deficit theory: The need for speed? Journal of Experimental $503 \quad$ Psychology. Human Perception and Performance 37:1957-1975.

504 McLean GMT, Stuart GW, Visser TAW, Castles A. 2009. The Attentional Blink in Developing $505 \quad$ Readers. Scientific Studies of Reading 13:334.

506 Moher D, Liberati A, Tetzlaff J, Altman DG, The PRISMA Group. 2009. Preferred Reporting 507 Items for Systematic Reviews and Meta-Analyses: The PRISMA Statement. PLoS Med $508 \quad 6: \mathrm{e} 1000097$.

509 Monsell S. 1996. Control of mental processes. In: Vicki B ed. Unsolved mysteries of the mind:

510 Tutorial essays in cognition. Hove, UK: Psychological Press Ltd, 93-148.

511 Niemi P, Näätänen R. 1981. Foreperiod and simple reaction time. Psychological Bulletin 89:133512 162.

513 Nobre A, Correa A, Coull J. 2007. The hazards of time. Current Opinion in Neurobiology 17:1-6. 
514 Olivers CNL, Hulleman J, Spalek T, Kawahara J, Di Lollo V. 2011. The Sparing Is Far From

515 Spurious: Reevaluating Within-Trial Contingency Effects in the Attentional Blink.

516 Journal of Experimental Psychology: Human Perception and Performance 37:396-408.

517 Potter MC, Chun MM, Banks BS, Muckenhoupt M. 1998. Two attentional deficits in serial target

518 search: the visual attentional blink and an amodal task-switch deficit. Journal of

519 Experimental Psychology. Learning, Memory, and Cognition 24:979-992.

520 Raymond JE, Shapiro KL, Arnell KM. 1992. Temporary suppression of visual processing in an

521 RSVP task: an attentional blink? Journal of Experimental Psychology. Human Perception

$522 \quad$ and Performance 18:849-860.

523 Roach NW, Edwards VT, Hogben JH. 2004. The tale is in the tail: An alternative hypothesis for

524 psychophysical performance variability in dyslexia. Perception 33:817 - 830.

525 La Rocque CL, Visser TAW. 2009. Sequential object recognition deficits in normal readers.

$526 \quad$ Vision Research 49:96-101.

527 Rogers RD, Monsell S. 1995. Costs of a predictable switch between simple cognitive tasks.

$528 \quad$ Journal of Experimental Psychology: General 124:207-231.

529 Rohatgi A. 2014. Version 3.3 of WebPlotDigitizer. ZENODO.

530 Rosenthal R. 1979. The file drawer problem and tolerance for null results. Psychological Bulletin $531 \quad 86: 638-641$.

532 Schneps MH, Thomson JM, Sonnert G, Pomplun M, Chen C, Heffner-Wong A. 2013. Shorter 533 Lines Facilitate Reading in Those Who Struggle. PLoS ONE 8:e71161.

534 Stuart GW, McAnally KI, Castles A. 2001. Can contrast sensitivity functions in dyslexia be 535 explained by inattention rather than a magnocellular deficit? Vision Research 41:3205$536 \quad 3211$. 
537 Tallal P. 1976. Rapid auditory processing in normal and disordered language development.

$538 \quad$ Journal of Speech \& Hearing Research 19:561-571.

539 Tang MF, Badcock DR, Visser TAW. 2013. Training and the attentional blink: Limits overcome 540 or expectations raised? Psychonomic Bulletin \& Review:1-6.

541 Valdois S, Bosse M-L, Ans B, Carbonnel S, Zorman M, David D, Pellat J. 2003. Phonological

542 and visual processing deficits can dissociate in developmental dyslexia: Evidence from $543 \quad$ two case studies. Reading and Writing 16:541-572.

544 Viechtbauer W. 2010. Conducting Meta-Analyses in R with the metafor Package. Journal of $545 \quad$ Statistical Software 36.

546 Visser TAW, Boden C, Giaschi DE. 2004. Children with dyslexia: evidence for visual attention 547 deficits in perception of rapid sequences of objects. Vision Research 44:2521-2535.

548 Ziegler JC. 2008. Better to lose the anchor than the whole ship. Trends in Cognitive Sciences $549 \quad 12: 244-245$. 
1

Fig 1 - Four patterns of attentional blink (AB) performance

Second target performance, given that the first target was correctly reported, as a function of the inter-target interval. The solid line depicts a standard AB performance. The dashed lines depict atypical $A B$ performance related to deeper (panel A), longer (B), and deeper and longer $A B$ effects $(C)$, as well as lower overall accuracy $(D)$.
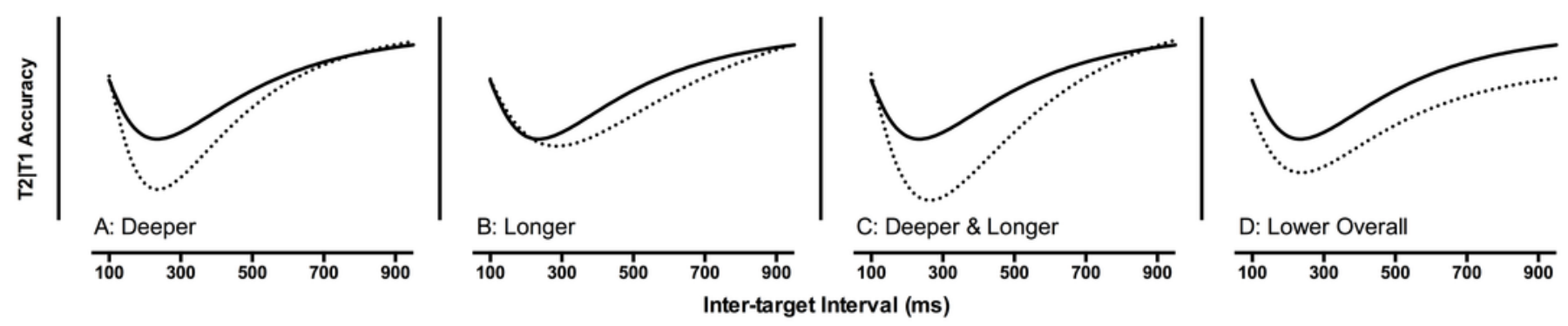


\section{2}

Fig 2 - Forest plot of the group difference in overall T2|T1 accuracy by experiment

Data point size is based upon the inverse of the variance (i.e. larger points correspond to smaller variance). T2|T1 denotes T2 accuracy calculated for those trials in which T1 was correctly reported.

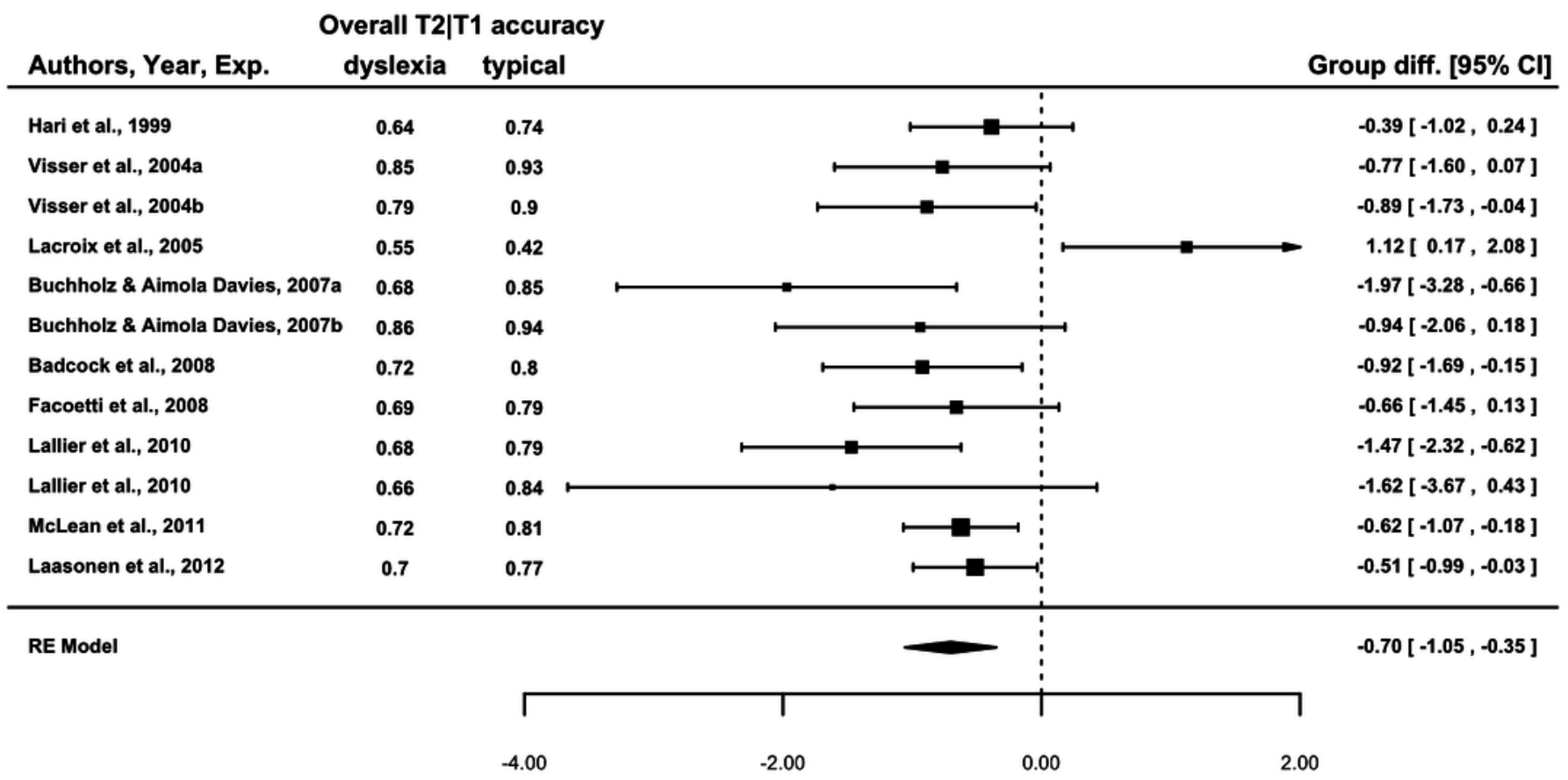

Group difference (Cohen's d) 


\section{3}

Fig 3 - Scatter plots and linear regression fits for the Group Difference (Cohen's d, yaxis) again pre-RSVP time (ms, panel A) and T2 time difference relative to T1 (ms, panel B).

Data point size is based upon the inverse of the variance (i.e. larger points correspond to smaller variance), solid lines are regression fits, and dashed lines are $95 \%$ confidence intervals.
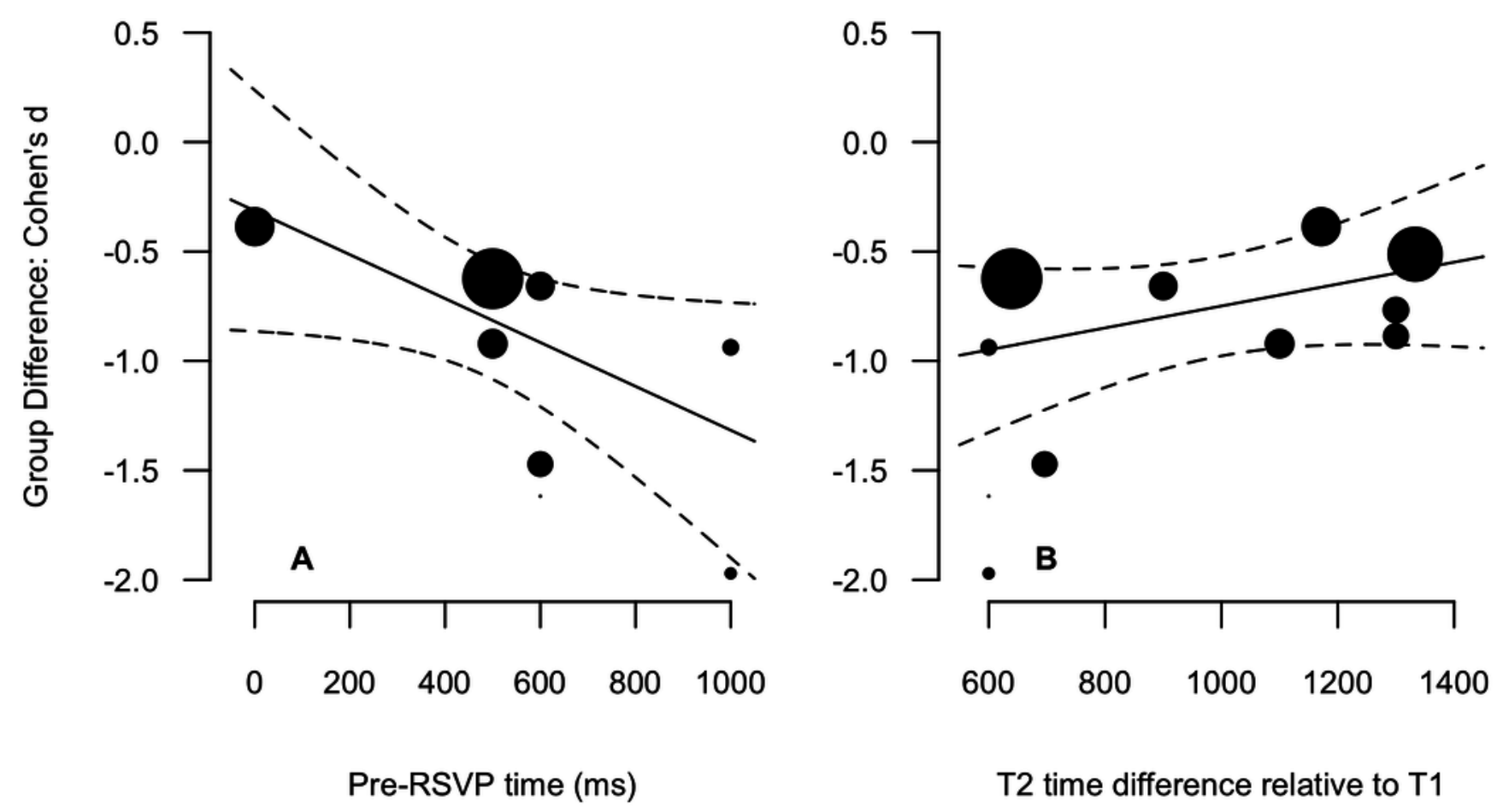

T2 time difference relative to $\mathrm{T} 1$ 


\section{4}

Fig 4 - Scatter plot and linear regression fit between the Group Difference (Cohen's d, yaxis) and Overall mean $\mathrm{T} 2$ accuracy (mean of the two groups).

Data point size is based upon the inverse of the variance (i.e. larger points correspond to smaller variance), solid lines are regression fits, and dashed lines are 95\% confidence intervals.

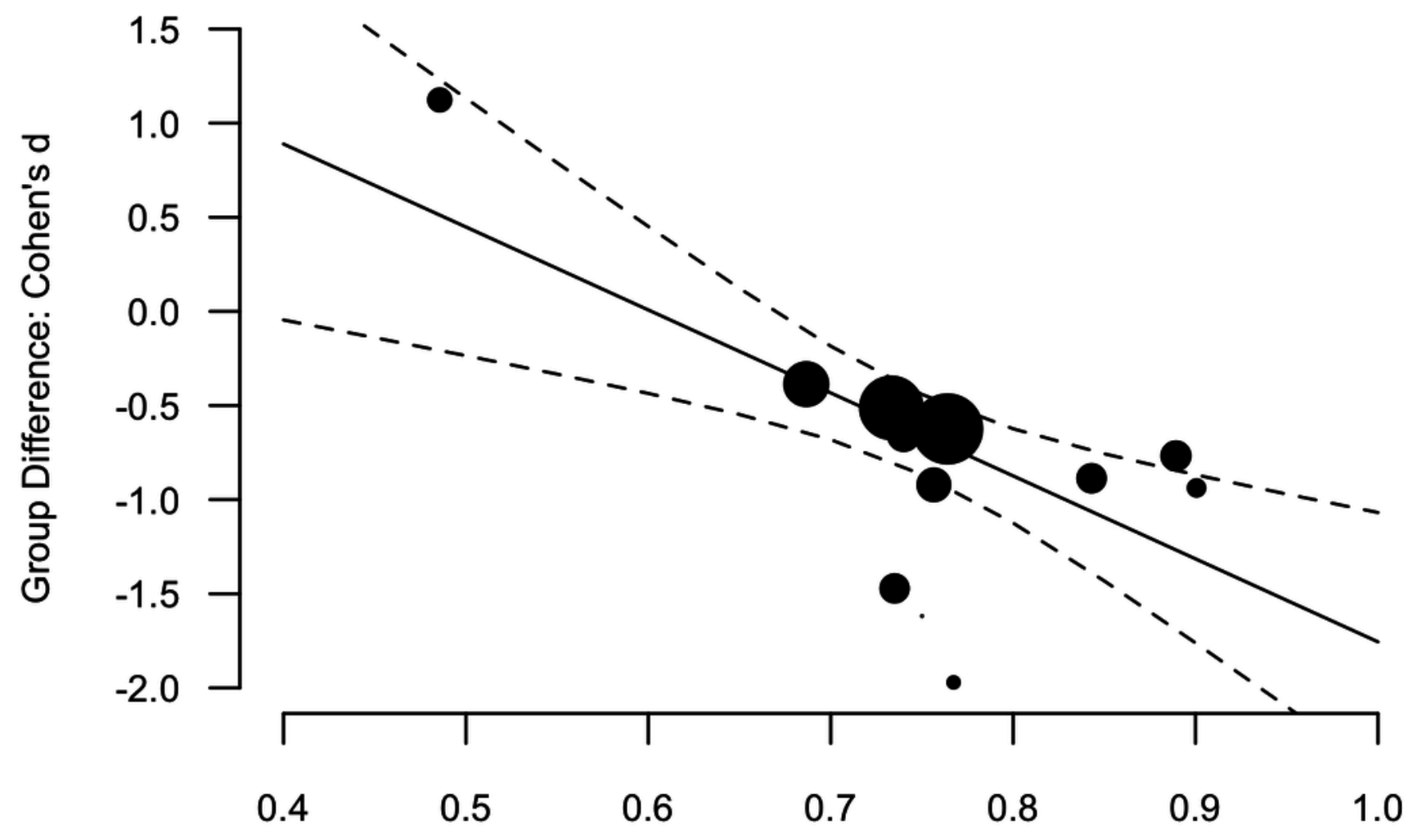

Overvall mean T2 accuracy 


\section{Table $\mathbf{1}_{\text {(on next page) }}$}

Parameter descriptive statistics, normality tests, and values for each experiment on the attentional blink and dyslexia

Descriptive statistics are based on $n=11$ experiments, excluding Lacroix et al. (2005) and McLean et al. (2010), except for the Pre-RSVP time variable that is based on $n=8$ experiments, excluding experimental tasks initiated with a key press. SOA = Stimulus Onset Asynchrony, $\mathrm{T} 1 / \mathrm{T} 2=$ first/second target, $\mathrm{T} 2$ time difference is relative to $\mathrm{T} 1$ temporal position, Group Difference is Cohen's d in standard deviation units, $* * p<.01, * p<.05$ 


\begin{tabular}{|c|c|c|c|c|c|c|c|c|c|c|}
\hline Statistic & & & $\begin{array}{c}\text { Pre-RSVP } \\
\text { time }^{\wedge}\end{array}$ & SOA & $\begin{array}{c}\text { T1 time } \\
\text { difference }\end{array}$ & $\begin{array}{c}\text { T2 time } \\
\text { difference }\end{array}$ & $\begin{array}{l}\text { RSVP time } \\
\text { difference }\end{array}$ & $\begin{array}{c}\text { Total } \\
\text { Identities }\end{array}$ & $\begin{array}{c}\text { Task } \\
\text { Complexity } \\
\end{array}$ & $\begin{array}{c}\text { Group } \\
\text { Difference }\end{array}$ \\
\hline Mean (SD) & & & $600(316)$ & $105.67(10)$ & $621.99(353)$ & $930.92(315)$ & $1179.07(854)$ & $13.82(7)$ & $1.64(1)$ & $-0.98(0)$ \\
\hline Median (IQR) & & & $600(200)$ & $100(6.55)$ & $424(593.25)$ & $900(615.95)$ & $958.5(737.5)$ & $12(9.5)$ & $1(2.5)$ & $-0.89(0.56)$ \\
\hline Min & & & 0 & 100 & 125 & 600 & 0 & 6 & 0 & -1.97 \\
\hline Max & & & 1000 & 133 & 1066 & 1333 & 3066 & 25 & 3 & -0.39 \\
\hline Shapiro-Wilk & & & 0.87 & $0.63 * *$ & $0.85^{*}$ & $0.83^{*}$ & 0.9 & 0.88 & $0.77 * *$ & 0.9 \\
\hline Authors & Year & Experiment & & & & & & & & \\
\hline Hari et al. & 1999 & 1 & 0 & 106.5 & 958.5 & 1171.5 & 958.5 & 25 & 3 & -0.39 \\
\hline Visser et al. & 2004 & 1 & key press & 100 & 300 & 1300 & 1600 & 6 & 0 & -0.77 \\
\hline Visser et al. & 2004 & 2 & key press & 100 & 300 & 1300 & 1600 & 6 & 1 & -0.89 \\
\hline Lacroix et al. & 2005 & 1 & 1000 & 100 & 400 & 700 & 0 & 10 & 1 & 1.12 \\
\hline $\begin{array}{l}\text { Buchholz \& Aimola } \\
\text { Davies }\end{array}$ & 2007 & 1 & 1000 & 100 & 400 & 600 & 0 & 9 & 1 & -1.97 \\
\hline $\begin{array}{l}\text { Buchholz \& Aimola } \\
\text { Davies }\end{array}$ & 2007 & 2 & 1000 & 100 & 400 & 600 & 0 & 17 & 0 & -0.94 \\
\hline Badcock et al. & 2008 & 1 & 500 & 100 & 900 & 1100 & 1600 & 20 & 3 & -0.92 \\
\hline Facoetti et al. & 2008 & 1 & 600 & 100 & 125 & 900 & 925 & 9 & 0 & -0.66 \\
\hline Lallier et al. & 2010 & 1 & 600 & 116 & 928 & 696 & 928 & 12 & 3 & -1.47 \\
\hline Lallier et al. & 2010 & 2 & 600 & 100 & 1040 & 600 & 800 & 12 & 3 & -1.62 \\
\hline McLean et al. & 2010 & 1 & 500 & 106.6 & 424 & 639.6 & 1492.4 & 11 & 1 & -0.79 \\
\hline McLean et al. & 2011 & 1 & 500 & 106.6 & 424 & 639.6 & 1492.4 & 11 & 1 & -0.62 \\
\hline Laasonen et al. & 2012 & 1 & key press & 133.3 & 1066.4 & 1333 & 3065.9 & 25 & 3 & -0.51 \\
\hline
\end{tabular}


Table 2 (on next page)

Correlations coefficients (Pearson: Spearman) between variables of the attentional blink experiments on developmental dyslexia $(n=11)$.

${ }^{\circ}$ non-normally distributed variable; ${ }^{\wedge} \mathrm{n}=8 ; \mathrm{SOA}=$ Stimulus Onset Asynchrony; $\mathrm{T} 1 / \mathrm{T2}=$ first/second target; rel $\mathrm{T} 1=$ relative to $\mathrm{T} 1 ; * * \mathrm{p}<.01, * \mathrm{p}<.05$ 


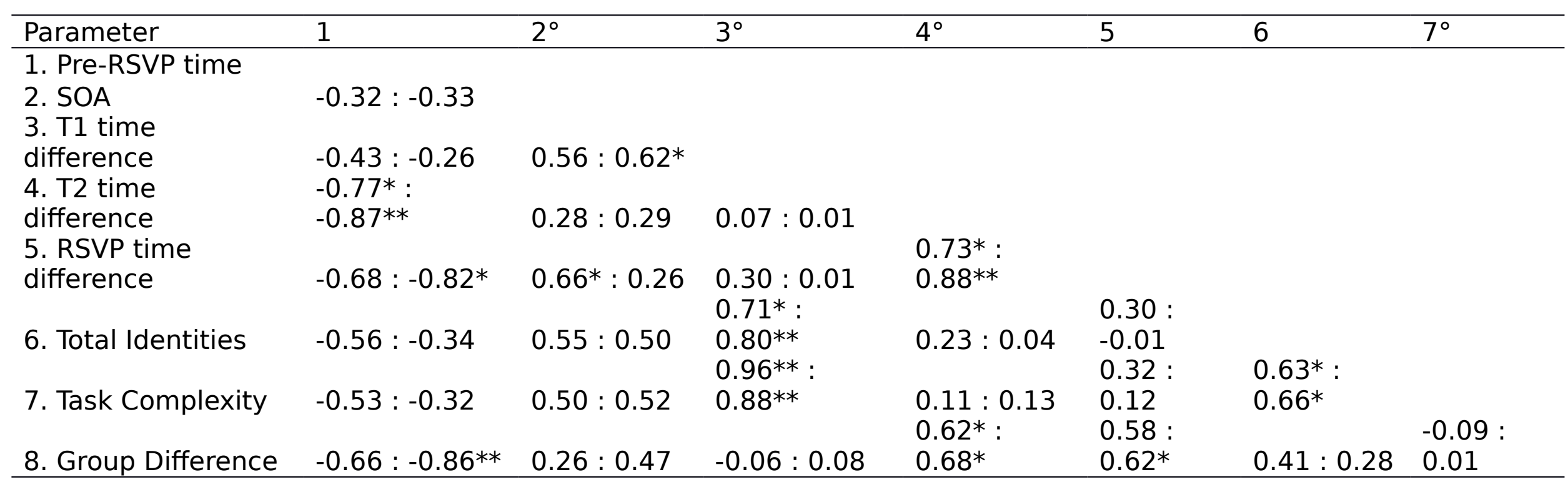

